\title{
Análisis de la gestión institucional en las unidades académicas de la Policia Nacional - Perú
}

\section{Analysis of institutional management in the academic units of the National Police - Perú}

Emigdio Melquiades Zavaleta Cabrera

0000-0002-2359-0483

Universidad Cesar Vallejo, Lima, Perú.

ezavaleta1804@gmail.com

Recibido: $04 / 012021$

Aceptado: 20/02/2021

Pub7icado: 17/03/2021

\section{Resumen}

La Gestión institucional constituye un aspecto de gran significación para la formación de nuevas generaciones de educandos de un país, verbigracia, si se trata de la formación de los futuros policías que son puestos al servicio de la sociedad para garantizar los derechos ciudadanos. En tal sentido, se presenta un estudio cuyo objetivo es analizar la gestión institucional en las unidades académicas de la Policía Nacional del Perú (UA-PNP); para lo cual se utilizó el método mixto, compuesta por un análisis documental y entrevistas a asesores, funcionarios y alumnos destacados del sistema educativo policial, para luego aplicar un cuestionario a los alumnos próximos a egresar de las unidades académicas. Los hallazgos evidencian que existen serias deficiencias en la gestión institucional, lo cual es producto de la falta de planificación, aplicación inadecuada de la estrategia y uso ineficiente de los recursos, que repercuten en la calidad de la formación policial, el buen servicio a la sociedad y la buena imagen institucional. Se concluye, así, que la gestión institucional es una herramienta importante para impulsar la conducción de la institución hacia el logro de los objetivos y metas organizacionales.

Palabras clave: Gestión institucional; unidades académicas; estrategia; imagen; Perú.

\begin{abstract}
Institutional management constitutes an aspect of great significance for the training of new generations of students in a country, for example, if it is about the training of future police officers who are placed at the service of society to guarantee citizen rights. In this sense, a study is presented whose objective is to analyze the institutional management in the academic units of the National Police of Peru (UA-PNP); For which the mixed method was used, consisting of a documentary analysis and interviews with advisers, officials and outstanding students of the police education system, to then apply a questionnaire to students close to graduating from academic units. The findings show that there are serious deficiencies in institutional management, which is the product of lack of planning, inadequate application of the strategy and inefficient use of resources, which have an impact on the quality of police training, good service to society and the good institutional image. It is concluded, thus, that institutional management is an important tool to promote the conduction of the institution towards the achievement of organizational objectives and goals.
\end{abstract}

Keywords: Institutional management; academic units; strategy; image; Peru. 


\section{Introducción}

Uno de los grandes desafíos a los que se enfrentan las instituciones, es el manejo de sus recursos para lograr los objetivos. Siguiendo a Serkan et al. (2019) la gestión institucional depende de las estrategias, manejo eficiente de los recursos y talento humano, factor importante en el logro de las metas organizacionales. Los recientes avances tecnológicos, hacen que las organizaciones enfrenten cambios sustanciales en sus estrategias y actividades de desarrollo (Besri y Boulmakoul, 2021).

En este contexto, y siendo la institución policial el principal instrumento social y fundamental para desarrollar y ejecutar políticas públicas de seguridad orientadas a combatir el delito, los centros de formación policial han de adoptar las estrategias para el uso eficiente de los recursos, cuyo impacto se vea reflejado en la efectividad y profesionalismo del accionar policial; resultados que no han logrado avances significativos, por las deficiencias presentadas a partir del nivel de formación. Nivel en donde la formación y el desarrollo de los colaboradores se debe gestionar adecuadamente, por tener una repercusión positiva en la seguridad social de un país (Lorek et al., 2020). No obstante, a pesar de los años transcurridos, aún existen deficiencias en la gestión como limitaciones en la organización, falta de planificación, que contribuyen a profundizar la crisis institucional (Álvarez et al., 2019). Esto cobra especial relevancia pues, según argumenta Bond (2020), es fundamental que la institución policial esté preparada para los cambios constantes y poder superar las ineludibles dificultades culturales, estructurales y políticos.

Estos graves problemas en la gestión, se ven agudizados por la pobre imagen pública que aqueja a la institución, donde los colaboradores juegan un rol importante y en ese mismo sentido, desarrollar sus habilidades, conocer las actividades que realizan, conlleva ejecutar bien la labor y buenos resultados en la gestión (Diaz et al., 2018). Otra repercusión es la percepción de inseguridad como problema medular de las sociedades de la posmodernidad, por el aumento de la violencia criminal que, según 448 líderes de opinión y periodistas de catorce países Latinoamericanos y el Caribe, es uno de los principales problemas que asediaron la región, con una tasa de ocho homicidios por cada 100,000 habitantes a nivel mundial y 24,5 homicidios por cada 100,00 habitantes en América Latina (Quintero, 2020). Es fundamental desarrollar estrategias y herramientas en la gestión, para el mejor desempeño de sus colaboradores y lograr en este mundo globalizado, una posición competitiva de la organización (Vera y Blanco, 2019).

En ese orden de ideas, el análisis de la gestión institucional responde a un proceso de reflexión respecto a las debilidades del actual sistema de formación policial y las consecuencias negativas que estas generan en el desempeño de la Policía Nacional, y por ende en la sociedad; y la necesidad de una gestión de cambio que permita al país, contar con policías debidamente formados $\mathrm{y}$ capacitados para enfrentar los problemas de la sociedad. Se requiere de las condiciones adecuadas para el desarrollo de los procesos de formación, preocupación constante en la institución policial para generar el cambio de las organizaciones policiales (Willis et al., 2020).

En el Perú, en las últimas décadas, como estrategia para enfrentar el accionar delictivo, el gobierno aperturó a nivel nacional 27 UA-PNP. Decisión que devino en una deficiente formación policial; se observan falencias en la gestión como: falta de laboratorios, campos deportivos, piscinas, galerías de tiro, centros de salud; en plataforma tecnológica, importante y prioritaria en la gestión para mejorar la capacidad de respuesta organizacional (Cadena et al., 2018). Las exigencias en seguridad cada vez son mayores y se requiere un policía más integral, mejor formado (Dussan et al., 2016). Este modelo de gestión, originó una estructura organizacional frondosa, ineficiente e ineficaz, lenta y burocrática en la toma de decisiones. Zapata y Canet (2012) refieren que, cuando existe complejidad en el trabajo, se deben descomponer estas en múltiples tareas, altamente especializada.

Existe poco interés de las instituciones policiales por promover desde su interior estudios desde las ciencias sociales (Barrera, 2016). Se hace necesario, desde las unidades académicas, comprender la gestión institucional como un proceso para lograr resultados: las metas y objetivos organizacionales, haciendo más eficiente y eficaz la institución, realizando una verdadera transformación de los estándares de funcionamiento, fortalecimiento y desarrollo institucional, una formación policial de calidad para responder a las expectativas de la ciudadanía, que exige de habilidades, competencias 
profesionales y efectividad en el desempeño de las funciones.

En ese sentido, cumplir con las políticas de Estado del Acuerdo Nacional relacionado con democracia y estado de derecho, y Estado eficiente, transparente y descentralizado, considerados en el Plan Estratégico de la Policía Nacional, articulado con el Plan Bicentenario, documento orientador del Sistema Nacional de Planeamiento Estratégico. La siguiente investigación buscó analizar la relevancia de la gestión institucional en las UA-PNP, en el logro de las metas y objetivos organizacionales.

\section{Marco teórico}

El mundo de hoy evoluciona constantemente y demanda de las organizaciones educativas, la delineación de proyectos más eficientes que les permita afrontar los desafíos que exige el desarrollo, las demandas de las personas y la sociedad (Oseda et al., 2020). Se deben formular las estrategias como respuestas al continuo cambio de las organizaciones para adaptarse mejor a la globalización; a los avances científicos y tecnológicos, donde los conocimientos quedan obsoletos con mayor rapidez (Palomeque y Ruiz, 2019).

Por lo tanto, se hace necesario comprender la gestión institucional con elementos centrados en el proceso administrativo como la planeación, organización, dirección y control, tendiente a producir un resultado en particular, conforme lo definiera la Escuela Operacional de Peter Drucker (Chiavenato, 2006). Un nuevo concepto para proponer estrategias en la toma de decisiones, para mejorar las funciones de la institución a través del liderazgo en los procesos de anticipación, transformación e innovación, en respuesta a contextos turbulentos; a diferencia del concepto de administración, donde simplemente se cumplen objetivos establecidos en los marcos normativos (De Donini y Donini, 2003).

Torres (2014) define la gestión como "el conjunto de acciones integradas para el logro de un objetivo a cierto plazo; es la acción principal de la administración y eslabón intermedio entre la planificación y los objetivos concretos que se pretenden alcanzar" (p. 57). Las manifestaciones de la gestión institucional existen a partir de la escuela clásica de la administración, con los pensamientos mecanicista de Taylor y anatómico de Fayol, que hicieron aportes importantes en la gestión, como el énfasis en las tareas con el empleo máximo del personal y la estructura, y proceso administrativo basado en los principios de la división del trabajo, autoridad, responsabilidad, disciplina, unidad de mando y dirección. Los estudios de Max Weber, propone un modelo de gestión organizacional basado en las normas y reglamentos, con un estrecho control de sus actividades bien definidas, para lograr las metas y objetivos organizacionales (Tejada et al., 2020).

El modelo de gestión burocrático que propone Weber, se utiliza para referirse principalmente a la administración pública, utilizándose actualmente este concepto de manera peyorativa en referencia a la pérdida de tiempo e ineficacia (Perea y Rojas, 2019). La organización policial responde al paradigma burocrático weberiano, por la forma como están dispuestos los niveles de organización. El director se ubica en la cúspide de la estructura organizacional y en la base, los colaboradores que prestan el servicio a la sociedad; se gobierna bajo el principio de jerarquía, y en todos sus ámbitos sometidos al derecho público y centralización en la toma de decisiones. La gestión está orientada primordialmente al cumplimiento de la legalidad y no a la eficiencia en el uso de los recursos públicos.

En la tabla 1, se hace una discriminación de los diferentes aspectos y dimensiones de la gestión institucional y que por la naturaleza de la investigación y criterio del investigador tomaremos en consideración integrándolas en las acciones más relevantes del proceso administrativo, agrupándolas dentro de la planeación, la organización, la dirección y el control, que permitirá hacer el análisis de la gestión institucional en las UA-PNP. 
Tabla 1.

Dimensiones de la gestión institucional.

\begin{tabular}{|c|c|c|}
\hline Autores & Dimensiones según autores & $\begin{array}{l}\text { Dimensiones de la } \\
\text { Investigación }\end{array}$ \\
\hline $\begin{array}{l}\text { Pacheco, Castañeda y Caicedo } \\
\text { (2002) }\end{array}$ & $\begin{array}{l}\text { Objetivos } \\
\text { Estrategia } \\
\text { Tecnología } \\
\text { Estructura } \\
\text { Métodos organizativos } \\
\text { Cultura Empresarial } \\
\text { Personalidad de los miembros de la } \\
\text { organización }\end{array}$ & \multirow{4}{*}{$\begin{array}{l}\text { Planificación: } \\
\text { Objetivos } \\
\text { Políticas } \\
\text { Estrategia } \\
\text { Eficiencia } \\
\text { Eficacia } \\
\text { Organización: } \\
\text { Estructura } \\
\text { Personas } \\
\text { Procesos } \\
\text { Recursos } \\
\text { Tecnología } \\
\text { Dirección: } \\
\text { Liderazgo } \\
\text { Control } \\
\text { Resultados en las personas } \\
\text { Resultado en los clientes } \\
\text { Resultados en la sociedad }\end{array}$} \\
\hline Molina (2014) & $\begin{array}{l}\text { Estrategia } \\
\text { Estructura } \\
\text { Métodos } \\
\text { Prácticas directivas } \\
\text { Instrumentos } \\
\text { Herramientas } \\
\text { Cultura } \\
\text { Personalidad } \\
\end{array}$ & \\
\hline Gonzales y Leguía (2015) & $\begin{array}{l}\text { Recursos } \\
\text { Resultados } \\
\text { Eficiencia } \\
\text { Eficacia } \\
\end{array}$ & \\
\hline Chiavenato (2006) & $\begin{array}{l}\text { Estrategia } \\
\text { Operatividad } \\
\text { Recursos } \\
\text { Políticas } \\
\text { Objetivos } \\
\text { Ambiente }\end{array}$ & \\
\hline
\end{tabular}

\section{Metodología}

Se presenta una investigación con enfoque mixto cuyo diseño corresponde a la triangulación concurrente (DITRIAC), aplicado en la parte de recolección, análisis e interpretación de datos, que implica, corroborar los resultados obtenidos y dar una validación cruzada entre los datos cualitativos y cuantitativos, aprovechando las ventajas de cada método para minimizar sus debilidades (Hernández et al., 2014). El enfoque mixto se fundamenta en el pragmatismo, que tiene como premisa, convocar a varios modelos mentales en el mismo espacio de búsqueda, para fines de un diálogo respetuoso y que los enfoques se nutran entre si (Greene, 2008).

Participantes. Para los datos cuantitativos, se obtuvo una muestra representativa no probabilística o muestras dirigidas, de 30 alumnos de las 27 unidades académicas a nivel nacional, seleccionados en consideración al criterio del tiempo de formación policial (próximos a egresar) y poseer una mejor percepción de la realidad problemática. La ventaja desde la óptica cuantitativa, es su utilidad para diseños que requieren una cuidadosa y controlada elección sobre los casos con características descritas en el objetivo de la investigación, que requieren no tanto una representatividad de los elementos de la población (Hernández et al., 2014). Han servido para corroborar los resultados obtenidos de las entrevistas y análisis documental.

Para los datos cualitativos, se utilizó un muestreo por conveniencia, seleccionándose a siete sujetos informantes: tres asesores, dos funcionarios y dos alumnos del sistema educativo policial en el nivel de formación. La decisión respecto a la muestra no busca la representatividad estadística, sino información confiable bajo el criterio del "informante calificado", en función de su relevancia con el tema y objetivo del estudio (Hernández et al., 2014). Por último, para el análisis documental se seleccionaron 20 documentos que convergen con el objetivo de la investigación, cuya búsqueda se realizó en línea utilizando el término gestión institucional en las escuelas de la PNP o haciendo mención a alguna de las unidades de significado relevante (USR). 
Técnica de recolección de datos. Encuesta a los alumnos de las UA-PNP para datos cuantitativos, con el propósito de conocer sus percepciones en relación al objeto de estudio, utilizándose el cuestionario, con catorce preguntas, empleando la escala de medición de actitudes tipo Likert, cuya validez fue sometida a juicio de tres expertos y la confiabilidad al coeficiente de Alfa de Cronbach, en una prueba piloto con resultado de 0,92 , altamente confiable, según interpretación de Valderrama (2013). Para datos cualitativos, la técnica de la entrevista semiestructurada, utilizándose la guía de entrevistas aplicada a los participantes; y, para el análisis o investigación documental, los documentos como instrumentos acordes a los objetivos propuestos.

Procedimiento de análisis de datos. Para los datos cualitativos producto de las entrevistas, se llevó a cabo el protocolo de análisis fenomenológico que proponen Molla et al. (2010), efectuando a través de una matriz, las acciones siguientes: codificación del discurso de los participantes del 1 al 7 (P1, P2,...P7); transcripción de las entrevistas; elaboración de las unidades de significado general con las relecturas de cada uno de ellos; agrupación en unidad de significado de aspectos relacionados con el tema de investigación; selección de USR; agrupación en categorías con características comunes y se concluye con las conclusiones, resaltándose los aspectos más importantes obtenidos en la investigación. El mismo procedimiento para el análisis documental, codificándose previamente los documentos del 1 al 20 (D1, D2,...D20). Los datos cuantitativos fueron obtenidos de la aplicación del software estadístico SPSS.

Limitaciones del estudio. Teniendoen consideración que la policía es una institución relevante y funcional para el Estado y el régimen político, es importante resaltar que, si bien la metodología permite alcanzar los objetivos planteados, es necesario que los hallazgos y resultados obtenidos, sean abordados con un mayor nivel de profundidad en futuras investigaciones.

Aspectos éticos. El estudio se desarrolló en el contexto policial, donde la información es estrictamente reservada y confidencial, siendo autorizado por la Dirección de la Escuela Nacional deFormaciónProfesionalPolicial.Los participantes accedieron en forma voluntaria, llevando a cabo un procedimiento de consentimiento informado, consistente en dar a conocer los objetivos y características del estudio.

\section{Resultados}

Los participantes de este estudio son asesores y funcionarios del sistema educativo policial, que conocen la gestión institucional y la realidad de las UA-PNP; también alumnos calificados con conocimiento en el tema de investigación. A partir del discurso de los participantes y el análisis documental, como resultado del trabajo de campo y acorde con los métodos empleados, en la tabla 2 se caracteriza la información de acuerdo a cuatro grandes categorías: planificación, organización, dirección y control.

Tabla 2.

Caracterización de la categoría gestión institucional.

\begin{tabular}{|c|c|c|c|}
\hline \multirow{3}{*}{$\begin{array}{l}\text { Unidades de } \\
\text { Significado } \\
\text { Relevante - USR }\end{array}$} & FUEN & & \multirow{3}{*}{ Caracterización } \\
\hline & Entrevistas & Documentales & \\
\hline & \multicolumn{2}{|c|}{ Planificación } & \\
\hline Planificación & No hubo planificación & No hay descripción específica & No hubo planificación \\
\hline Estrategia & No fue la más adecuada & No hay descripción específica & No fue la adecuada \\
\hline Objetivos & No se cumplen los objetivos & No se cumplen & No se cumplen \\
\hline $\begin{array}{l}\text { Ineficiencia e } \\
\text { ineficacia }\end{array}$ & $\begin{array}{l}\text { No existe eficiencia ni eficacia en la } \\
\text { gestión }\end{array}$ & No hay evidencia & No existe \\
\hline $\begin{array}{l}\text { Rotación de } \\
\text { directores }\end{array}$ & $\begin{array}{l}\text { Cada uno o dos años conforme la } \\
\text { norma }\end{array}$ & $\begin{array}{l}\text { Anualmente de acuerdo a los } \\
\text { Cambios Generales de } \\
\text { Colocación }\end{array}$ & $\begin{array}{l}\text { De acuerdo a las } \\
\text { normas }\end{array}$ \\
\hline $\begin{array}{l}\text { Periodo de } \\
\text { formación }\end{array}$ & No se cumple conforme a Ley & No se cumple & No se cumple \\
\hline $\begin{array}{l}\text { Formación de } \\
\text { directores }\end{array}$ & $\begin{array}{l}\text { No tienen formación ni preparación } \\
\text { en gestión educativa }\end{array}$ & No está documentada & $\begin{array}{l}\text { Sin formación } \\
\text { académica }\end{array}$ \\
\hline
\end{tabular}




\begin{tabular}{|c|c|c|c|}
\hline $\begin{array}{l}\text { Unidades macro } \\
\text { regionales }\end{array}$ & $\begin{array}{l}\text { Solo debe haber unidades macro } \\
\text { regionales }\end{array}$ & $\begin{array}{l}\text { Centralizar macro } \\
\text { regionalmente }\end{array}$ & $\begin{array}{l}\text { Debe haber UA-PNP } \\
\text { macro regionales }\end{array}$ \\
\hline \multicolumn{4}{|c|}{ Organización } \\
\hline Excesivas UA-PNP & Excesivas UA-PNP & Racionalizar UA-PNP & Excesivas UA-PNP \\
\hline Recursos & Carecen de todo & Falta de recursos & Falta de recursos \\
\hline $\begin{array}{l}\text { Requisitos de } \\
\text { funcionamiento }\end{array}$ & $\begin{array}{l}\text { Sin requisitos mínimos de } \\
\text { funcionamiento }\end{array}$ & Formulada en documentos & $\begin{array}{l}\text { UA-PNP sin requisitos } \\
\text { de funcionamiento }\end{array}$ \\
\hline Infraestructura & Carecen de todo & Inadecuada infraestructura & Inadecuada \\
\hline $\begin{array}{l}\text { Plataforma } \\
\text { tecnológica }\end{array}$ & $\begin{array}{l}\text { No acorde con el avance científico } \\
\text { tecnológico }\end{array}$ & Deficiente equipamiento & Deficiente \\
\hline Modelo de gestión & $\begin{array}{l}\text { No garantiza el desarrollo de las } \\
\text { actividades }\end{array}$ & No está documentada & Inadecuado \\
\hline $\begin{array}{l}\text { Estándares de } \\
\text { calidad }\end{array}$ & No cuentan con estándares de calidad & $\begin{array}{l}\text { UA-PNP sin estándares de } \\
\text { calidad }\end{array}$ & Sin estándares \\
\hline Docentes calificados & No se cuenta & Sin preparación pedagógica & No se cuenta \\
\hline \multicolumn{4}{|c|}{ Dirección } \\
\hline Toma de decisiones & No se han tomado decisiones & No se han tomado decisiones & $\begin{array}{l}\text { No se toman } \\
\text { decisiones }\end{array}$ \\
\hline Decisiones políticas & $\begin{array}{l}\text { UA-PNP creadas por cuestiones y } \\
\text { criterio político más que técnico }\end{array}$ & $\begin{array}{l}\text { UA-PNP creadas por cuestiones } \\
\text { políticas }\end{array}$ & $\begin{array}{l}\text { UA-PNP creadas } \\
\text { políticamente }\end{array}$ \\
\hline \multicolumn{4}{|c|}{ Control } \\
\hline $\begin{array}{l}\text { Inseguridad } \\
\text { ciudadana }\end{array}$ & $\begin{array}{l}\text { Alta percepción de inseguridad } \\
\text { ciudadana }\end{array}$ & Se incrementó & Alta percepción \\
\hline $\begin{array}{l}\text { Calidad en la } \\
\text { formación policial }\end{array}$ & No se ha recibido & $\begin{array}{l}\text { Escaza profesionalización. Mala } \\
\text { calidad de la formación policial }\end{array}$ & No se ha recibido \\
\hline Servicio policial & $\begin{array}{l}\text { Malas intervenciones policiales, mal } \\
\text { servicio a la sociedad. No se cubre las } \\
\text { expectativas de la ciudadanía }\end{array}$ & Deficiente & Deficiente \\
\hline
\end{tabular}

El gobierno central para enfrentar el accionar delictivo creó a nivel nacional 27 UA-PNP, sin la debida planificación. Sin embargo, este crecimiento en la organización no se evidencia en documento alguno, se fueron creando por cuestiones e intereses políticos, sin criterio técnico, con el apoyo de los gobiernos regionales y locales. No fue la estrategia más adecuada para formar policías con las competencias exigidas en el servicio policial; por lo tanto, no se cumplieron con las funciones establecidas en el marco jurídico y por ende con las metas y objetivos, existiendo ineficiencia e ineficacia en el manejo de los recursos.

Según la Ley de la Carrera y Situación del personal policial, anualmente los directores de la UA-PNP, son rotados de puesto, medida que imposibilita la especialización en el manejo de la gestión y dificulta la continuidad de políticas de mediano y largo plazo en el aspecto formativo. Un aspecto importante a tener en consideración, es que los directores son oficiales que han recibido formación policial. No tiene formación en gestión educativa, para conducir con éxito las unidades académicas, salvo algunas excepciones.
Según establece el régimen educativo policial, el tiempo de formación de los alumnos es de seis semestres o tres años, que en la práctica se reduce a solo año y medio; es decir, tres semestres académicos, egresando como Sub Oficiales, complementando su formación los días sábados o en forma virtual.

Se deben racionalizar las UA-PNP, debiendo existir unidades académicas macro regionales, grandes unidades que funcionen conforme las regiones policiales a nivel nacional, suprimiendo aquellas que no cuentan con los requisitos mínimos de funcionamiento y estándares de calidad. Se ha observado inadecuada infraestructura y falta de recursos, funcionan en locales cedidos en uso que no reúnen los requisitos mínimos de funcionamiento. Existe un deficiente equipamiento en plataforma tecnológica, a pesar de ser una prioridad en la gestión por el avance científico tecnológico del mundo moderno.

El modelo de gestión originó, además, una estructura organizacional frondosa, ineficiente e ineficaz, lenta y burocrática en la toma de 
decisiones. No existe un nivel jerárquico entre el director del sistema educativo y los directores de UA-PNP, siendo demasiado amplio el tramo de control administrativo. La lejanía de algunas unidades hace imposible contar con docentes calificados, asumiendo este rol improvisadamente, el personal policial asignado a dichas unidades.

Existe una alta percepción de inseguridad ciudadana. No se ha logrado calidad en la formación policial, repercutiendo en un deficiente servicio a la sociedad, teniendo en consideración que las exigencias en seguridad cada vez son mayores, se requiere un policía mejor formado con las competencias necesarias para afrontar con éxito la misión. Situación que no es coherente con la reforma policial para formar policías eficientes y eficaces; es contraproducente con la Ley general de educación, que considera inversión en infraestructura, mejor equipamiento y mínima inversión por alumno, conforme las exigencias técnico-pedagógicas de cada lugar; $\mathrm{y}$, a los avances científico tecnológico del mundo contemporáneo.

Un aspecto importante en el recojo de información, en la parte cuantitativa, es la percepción de la gestión institucional en los alumnos de las UAPNP, a través de encuestas, para contrastarlas con los resultados cualitativos, obtenidos producto de las entrevistas y análisis documental. En la tabla 3, se tienen los resultados que demuestran que el 10 y el $36.67 \%$ de los encuestados perciben que existe una deficiente y regular gestión institucional; el $53.33 \%$ perciben que existe buena gestión institucional en las UA-PNP.

Se puede deducir que, en las percepciones de los alumnos con respecto a la gestión institucional, el nivel predominante se encuentra en la opción regular y buena, lo cual es relativamente favorable por la gestión que realizan los directores en las UA-PNP.

Tabla 3.

Percepción de la gestión institucional.

\begin{tabular}{|c|c|c|c|c|c|}
\hline & & Frecuencia & Porcentaje & $\begin{array}{l}\text { Porcentaje } \\
\text { válido }\end{array}$ & $\begin{array}{l}\text { Porcentaje } \\
\text { acumulado }\end{array}$ \\
\hline \multirow[t]{4}{*}{ Válido } & Deficiente & 3 & 10,0 & 10,0 & 10,0 \\
\hline & Regular & 11 & 36,7 & 36,7 & 46,7 \\
\hline & Buena & 16 & 53,3 & 53,3 & 100,0 \\
\hline & Total & 30 & 100,0 & 100,0 & \\
\hline
\end{tabular}

En la tabla 4, se tienen los resultados que demuestran la percepción de los alumnos con respecto a la gestión institucional y sus dimensiones. En la dimensión planificación el 6.7 y $36.7 \%$ de los encuestados percibe que existe una deficiente y regular gestión en el manejo institucional, mientras que el $56.7 \%$ percibe como buena la gestión en las UA-PNP; en donde se puede deducir que el nivel predominante se encuentra en la opción regular y buena, lo cual es relativamente favorable por la gestión que realizan los directores de las UA-PNP.

En la dimensión organización, el 10 y $40 \%$ perciben como deficiente y regular la gestión institucional, mientras el 50\% percibe como buena la gestión; en donde se puede deducir que los niveles deficiente y regular, así como el bueno, tienen percepciones divididas, lo cual es relativamente favorable por la gestión que realizan los directores de las UA-PNP.

En la dimensión dirección el 26.7 y $33.3 \%$ perciben una deficiente y regular gestión, mientras que solo el $40 \%$, buena; el cual no es relativamente favorable por algunas falencias en la gestión de los directores, en el proceso de dirección en las UAPNP.

Por último, en la dimensión control el 20 y 37.7\% perciben deficiente y regular gestión, mientras que el $43.3 \%$ perciben como buena la gestión institucional; el cual no es relativamente favorable por algunas falencias en la gestión de los directores, en el proceso de control de las UA-PNP. 
Tabla 4.

Percepción de la gestión institucional en las dimensiones de las UA-PNP.

\begin{tabular}{llllll}
\hline \multirow{2}{*}{ Niveles } & Variables & \multicolumn{4}{l}{ Dimensiones } \\
\cline { 2 - 6 } & $\begin{array}{l}\text { Gestión } \\
\text { Institucional }\end{array}$ & \multicolumn{4}{l}{ Planificación } \\
& Organización & Dirección Control \\
\hline Deficiente & 10.0 & 6.7 & 10.0 & 26.7 & 20.0 \\
Regular & 36.7 & 36.7 & 40.0 & 33.3 & 36.7 \\
Buena & 53.3 & 56.7 & 50.0 & 40.0 & 43.3 \\
\hline Total & 100.0 & 100.0 & 100.0 & 100.0 & 100.0 \\
\hline
\end{tabular}

\section{Discusión}

El análisis de la información producto de las entrevistas, documentos y en cumplimiento al objetivo "analizar la gestión institucional en las unidades académicas de la Policía Nacional del Perú", ha permitido corroborar que:

En el proceso de planificación, el actual modelo de gestión institucional cuenta con excesivas UAPNP (27), creadas sin la debida planificación, sin sustento técnico, no fue la estrategia más adecuada, no permite el cumplimiento de los objetivos para asignar al servicio policías con una formación de calidad, que redunde en un excelente servicio a la comunidad. Cuando no se realiza el planeamiento estratégico, no se canalizan en una sola dirección los esfuerzos en el desarrollo de las actividades administrativas para cumplir los objetivos, como lo plantea Castro (2017). Es coincidente con la definición propuesta por Chávez (2019) quien señala que, se incrementan unidades académicas sin la debida planificación y estándares mínimos de funcionamiento y que las acciones definidas en su objetivo impiden desarrollar estrategias para combatir la inseguridad.

Asociado a la designación de directores sin la formación académica, Silva (2018) plantea que se debe proponer a Oficiales que respondan a los requerimientos que exigen los cargos para desarrollar con efectividad las funciones asignadas. Con relación al periodo de formación de los alumnos, la norma establece que debe ser tres años o seis semestres académicos; en la práctica esto se reduce a solo año y medio. Fernández (2015) sostiene que es una decisión política para mantener más policías en las calles y que se debe respetar su periodo de formación como elemento fundamental y que solo con policías bien formados se podrá enfrentar con efectividad la inseguridad ciudadana.
En esta línea, Gavilanes (2019) sustenta, que la escuela de formación de policías realiza sus actividades internas con la ausencia de un modelo administrativo que le permita tener una gestión eficiente; que existe una rotación constante del personal debido a que no poseenun perfil profesional acorde al puesto de trabajo, lo que provoca atrasos en los procesos internos; que existen miembros en la organización que son Oficiales de policías que no poseen las competencias y habilidades requeridas en el puesto. En ese sentido, tal como lo expresan Llanos et al. (2018), se deben establecer en las ciudades corporaciones grandes, en razón que el tamaño y las tecnologías permiten el establecimiento de estrategias y la elaboración de una estructura para el éxito organizacional.

En el proceso de organización, se debió potenciar aquellas que cumplen con los estándares de funcionamiento. Los recursos no se manejan correctamente, carecen de todo, de infraestructura y las que hay, no cuentan con los requisitos mínimos de funcionamiento; desarrollan sus actividades en locales inapropiados, cedidos en uso, que no beneficia en la calidad de formación; no tienen piscina, campos deportivos, galerías de tiro, biblioteca, tecnología, internet. Al tener muchas UA-PNP, se vuelven limitados para el desarrollo de las actividades académicas. Uno de los problemas de la gestión, es que no cuentan con la plataforma tecnológica para estar acorde con el avance científico tecnológico; no garantiza el desarrollo de las actividades dentro de los estándares de calidad.

En efecto, Castro (2017) sostiene que, el plan estratégico considera mejorar los procesos, implementar tecnologías que facilite acceso a internet, modernizar la administración del talento humano, implementar laboratorios de investigación, equipar la biblioteca, es situar a la organización como institución referente en 
gestión de la calidad, garantizando la formación de profesionales altamente capacitados, con infraestructura y espacios bien definidos para el ámbito de especialización profesional.

En ese orden de ideas, Pereyra (2020) manifiesta que, existen múltiples deficiencias en la formación policial, infraestructura, recursos logísticos, capacidad formativa para el cumplimiento de la función; planteando una infraestructura adecuada que contribuya a mejorar la problemática existente. La crisis es multidimensional y abarca desde la infraestructura hasta la calidad de la formación del personal policial; se deben generar estructuras organizacionales que respondan a la implementación de la estrategia, como las unidades macro regionales a las que se hace mención Dammert y Castañeda (2019).

El proceso de innovación tecnológica incluye un componente de gestión del cambio institucional desde abajo hasta arriba, basado en la capacidad de la policía de innovar para desarrollar habilidades o competencias para la solución de problemas desde la formación policial, que permita una fuerza pública más profesional, más innovadora y más analítica, con el fin de impactar en los índices de criminalidad del país (Villalobos, 2020).

En el proceso de dirección, no se ha tomado la decisión para disminuir el número de escuelas, creadas por cuestiones y criterio político, a pesar de los estudios realizados y sugerido a la alta dirección. Se tiene que coordinar con 27 directores de escuelas, dificultando que la toma de decisiones sea oportuna para solucionar los diferentes problemas en cada UA-PNP, que requieren de soluciones urgentes para lograr los objetivos.

En el proceso de control, se percibe con mayor rapidez una alta percepción de inseguridad ciudadana, se requieren nuevas estrategias, policías mejor formados. En el actual modelo de gestión los sub oficiales no han recibido una formación y educación de calidad; las deficientes intervenciones policiales, repercuten en un mal servicio a la sociedad. Percibe que la PNP no está cumpliendo sus funciones, y no cubre las expectativas de la ciudadanía; existe desconfianza.

Asociado a esto, Dammert (2019) plantea que los cambios en los procesos formativos han sido lentos, careciendo de centros académicos que puedan fortalecer la formación policial, existiendo iniciativas de cambio en la modernización y el equipamiento, así como la adquisición de nuevas tecnologías que permitan el control más efectivo del delito. La calidad del recurso humano policial es posiblemente el principal talón de Aquiles institucional, al no contar con requisitos básicos de educación ni capacitados en las mejores estrategias para controlar el delito.

Estas deficiencias en los procesos de la gestión institucional son coincidentes con la definición propuesta por Giraldo (2020) quien señala, que la gestión institucional a través del planeamiento, la organización, la dirección y el control, es uno de los factores más importantes para el cumplimiento de los objetivos y el éxito institucional. Los modelos de gestión analizados presentan limitaciones en los procesos académicos y administrativos dentro de su estructura de funcionamiento, no se evidencia el papel de la planeación estratégica, definiendo adecuar sus estructuras y modelos de gestión a los desafíos actuales (Yépez y Pozo, 2018).

\section{Corroboración de datos cuantitativos}

El análisis de la información producto de la aplicación del cuestionario, donde se puede apreciar que la variable gestión institucional tiene cuatro dimensiones, de manera general, se puede apreciar que el $53.33 \%$ de los encuestados poseen una percepción buena sobre la gestión institucional en las UA-PNP; el 36.67\% una percepción regular; $\mathrm{y}$, el $10 \%$ una percepción deficiente sobre la gestión institucional. Se puede deducir que el nivel predominante se encuentra en la opción regular y buena, el cual es relativamente favorable, por la función que realizan los directores de las UA-PNP.

Estos resultados en la gestión, son coherentes con los obtenidos en el análisis de los datos cualitativos, que refleja serias deficiencias en el proceso de planificación, organización, dirección y control. Tarrillo (2018) sostiene que, la formación profesional y la gestión institucional presentan una relación directa encontrándose las dos en niveles mínimos y deficientes; los problemas más preocupantes son las decisiones respecto al cambio tecnológico acorde con el avance científico, la ineficiencia en la labor profesional de los estudiantes, la falta de actualización de la estructura organizacional, sus manuales y los procesos; recomendando aplicar mejoras en la gestión institucional para la formación policial de los alumnos. 


\section{Conclusiones}

Existen serias deficiencias en la gestión institucional en las UA-PNP, en los procesos de planeación, organización, dirección y control:

En el proceso de planificación: falta de planificación; estrategia inadecuada; ineficiencia e ineficacia en la gestión; asignación de directores por el periodo de un año, sin formación en gestión educativa; incumplimiento de objetivos; periodo de formación de año y medio, contraproducente a la norma que establece tres años. En el proceso de organización: estructura frondosa con excesivas UA-PNP, creadas sin sustento técnico; falta de recursos; infraestructura no cuenta con los requisitos mínimos de funcionamiento; desarrollan actividades en locales inapropiados cedidos en uso; la distante ubicación de las UA-PNP no permite la captación de docentes calificados; no tienen piscina, campos deportivos, galerías de tiro, plataforma tecnológica; situación que no garantiza el desarrollo de las actividades dentro de los estándares de calidad.

En el proceso de dirección: se realizaron los estudios pertinentes y sugerido a la alta dirección reducir el número de UA-PNP creadas por decisiones políticas, a unidades macro regionales; dificultad en la toma de decisiones y el aprovechamiento de los recursos. Por último, en el proceso de control: alta percepción de inseguridad ciudadana; deficiente servicio policial, producto de la mala 62 calidad en la formación policial.

De la exposición precedente, es posible extraer como idea general, que la gestión institucional es una herramienta que tiene un rol relevante en el cumplimiento de las metas y objetivos de la organización, a partir de la aplicación de una buena estrategia, uso eficiente de los recursos y el talento humano.

\section{Referencias bibliográficas}

Álvarez,L.,Ponce,D.\&Gómez,C.(2019).Academic strategy for the implementation of the teaching management model and the method for vocational training at the Uniandes Quevedo University. Dilemas Contemporáneos: Educación, Política y Valores. https:// search.proquest.com/scholarly-journals/ academic-strategy-implementation-teaching/ docview $/ 2245649427 /$ se-2? accountid $=37408$
Barrera, J. (2016). Police studies in Latin America. Some theoretical and methodological limitations in their approach a direct target. Revista Criminalidad, 58(3), 119-132. https:// www.scopus.com/inward/record.uri? eid $=2$ s2.0-85018948594\&partnerID $=40 \& m d 5=71$ 653b10ac3456bbe6afc8f90cbbac5a

Besri, Z. \& Boulmakoul, A. (2021). An Intuitionist Fuzzy Method for Discovering Organizational Structures that Support Digital Transformation. Advances Intelligent and Computing, 1197 AISC, 331-338. https://doi. org/10.1007/978-3-030-51156-2 39

Bond, B. (2020). Organizational Changein an Urban Police Department: Innovating to Reform. Organizational Change in an Urban Police Department: Innovating to Reform, 1-193. https://doi.org/10.4324/9781315641225

Cadena, L., Filian, F., Maldonado, A. \& Torres, R. (2018). Technology and public management. Dilemas Contemporáneos: Educación, Politica y Valores. https://search.proquest. com/scholarly-journals/technology-publicmanagement/docview/2247184493/se2? accountid $=37408$

Castro, J. (2017). Planificación Estratégica de una unidad administrativa de la Policía Nacional del Perú para el periodo 2018-2030 [Tesis de Maestría] Lima, Perú. Universidad Cesar Vallejo.

Chávez, N. (2019). Limitaciones del capital humano de la Policía Nacional del Perú, como factor estratégico de competitividad, para la implementación de las estrategias de prevención del delito durante el año 2016 - 2017. [Tesis de Maestría]. Pontificia Universidad Católica del Perú.

Chiavenato, I. (2006). Introducción a la Teoría General de Administración. 7ma ed. México: McGraw-Hill/Interamericana Editores, S.A. de C. V.

Dammert, L. (2019). ¿Reformar sin gobernar? Desafíos institucionales de las policías en América Latina. Revista IUS, 13(44), 89-120. https://doi.org/10.35487/rius. v13i44.2019.444

Dammert, L. y Castañeda, M. (2019). ¿Marketing o efectividad? Policía comunitaria en Perú. Utopía y Praxis Latinoamericana, 24(2), 5167. https://doi.org/10.5281/zenodo.3344846

De Donini, A. \& Donini, A. (2003). La gestión universitaria en el siglo XXI. Desafíos de la sociedad del conocimiento a las políticas académicas y científicas. Documento de 
Trabajo $\mathrm{N}^{\circ}$ 107, Universidad de Belgrano. Argentina. $\quad$ http://repositorio.ub.edu.ar/ bitstream/handle/123456789/393/107 donini.pdf? sequence $=2$ \&isAllowed $=y$

Díaz, J., Núñez, L. y Cáceres, K. (2018). Influencia de las competencias gerenciales y la gestión por resultados en la imagen institucional. Difusión cultural y cientifica de la Universidad La Salle en Bolivia, 16(16), 169-197. http://www.scielo.org.bo/scielo. php? script $=$ sci arttext\&pid $=$ S2071-081X201 8000200010\&lng=es\&tlng=es.

Dussán, C., Serna, H. y Caliman, G. (2016). Pensamiento estratégico, el gran desafío para las fuerzas armadas de Colombia. strategic thinking, the great challenge for the colombian armed forces. Logos, Ciencia \& Tecnología, 7(2), 5-13. https://doi.org/10.22335/rlct. v7i2.152

Fernández, W. (2015). Egreso anticipado de los alumnos de las Escuelas de Educación Superior Técnico Profesional de la Policía Nacional del Perú 2012-2014 [Tesis de Maestría]. Pontificia Universidad Católica del Perú.

Gavilanes, M. (2019). Asignación de funciones en la Escuela de Formación de Policías CBOS Sócrates Arboleda Sanabria del Cantón Babahoyo. [Tesis título profesional]. Universidad Técnica de Babahoyo. Ecuador.

Giraldo, E. (2020). Plan de mejora en la gestión administrativa para promover la eficiencia operativa en la Marina de Guerra del Perú sede Piura-2019. [Tesis título profesional]. Universidad Cesar Vallejo. Perú.

Greene, J. (2008). Is Mixed Methods Social Inquiry a Distinctive Methodology?. Journal of Mixed Methods Research, 2(1), 7-22. https://doi.org/10.1177/1558689807309969

Hernández, R., Fernández C., y Baptista (2014). Metodología de la investigación. 5ta ed. México: McGraw-Hill/Interamericana Editores, S. A. de C. V.

Llanos, L., Rivas, L., Lambarry, F. y Trujillo, M. (2018). La eficacia de la policía estatal en México. Perfiles Latinoamericanos, 26(52), 1-26. https://doi.org/10.18504/p12652-0152018

Lorek,. M., Piecuch, T., Itrich, J., \& Minkina, M. (2020). Management of the police officers training system and the effectiveness of internal security. Journal of Security and Sustainability Issues, 9(4), 1337-1348. https:// doi.org/10.9770/jssi.2020.9.4(19)
Molla, R., Bonet, R. y Climent, C. (2010). Propuesta de análisis fenomenológico de los datos obtenidos en la entrevista. Universitas Tarraconensis. Revista de Ciències de l'Educació, 25(20), p. 113-133. https://doi. org/10.17345/ute.2010.1.643

Oseda, D., Mendivel, R. \& Durán, A. (2020). Potencial de innovación y gestión institucional en la Universidad Nacional de Cañete-Perú. Sophia, Colección de Filosofía de la Educación, (28), 207-236. https://doi. org/10.17163/soph.n28.2020.08

Palomeque, I. \& Ruiz, J. (2019). Impact of institutional management on the generation of scientific knowledge in higher education institutions. Espacios, 40(2), 14p. https:// www.revistaespacios.com/a19v40n02/ a19v40n02p14.pdf

Perea, L., \& Rojas, I. (2019). Modelos de gestión en instituciones hospitalarias. Revista Gerencia y Políticas de Salud, 18(36). https:// doi.org/10.11144/Javeriana.rgps18-36.mgih

Pereyra, A. (2020). Infraestructura para mejorar la eficiencia y cantidad de personal policial ante la creciente inseguridad ciudadana [Tesis título profesional] Universidad Señor de Sipán. Perú.

Quintero, S. (2020). Citizen security and community participation in Latin America. Revista Científica General José María Córdova, 18(29), 5-21. https://doi. org/10.21830/19006586.561 http://www. scielo.org.co/scielo.php?pid $=$ S1900-

Serkan, N., Kanan, S., Bayraktar, Özgü, Emiroğlu, O., \& Güneyli, A. (2019). Levels of readiness in total quality management. Amazonia Investiga, 7(16), 268-275.

Silva, R. (2018). El servicio policial ineficiente en las dependencias policiales, principalmente durante los primeros meses del año, que afecta la Seguridad Ciudadana y por ende el normal desarrollo de las actividades de los Ciudadanos. [Tesis de Maestría] Pontifica Universidad Católica del Perú.

Tarrillo, I. (2018). Propuesta de mejoras en la gestión institucional para la formación profesional de los estudiantes de la escuela técnica de suboficiales de la Policía Nacional del Perú, Reque-Chiclayo, 2017 [Tesis título profesional] Universidad Señor de Sipán. Perú.

Tejada, G., Rengifo, R., Boy, A. \& Rodríguez, J. (2020). Organizational structure and control in a bureaucratic management model. 
Revista Venezolana de Gerencia, 25(89), 66-78. $\quad$ https://doi.org/10.37960/revista. v25i89.31385

Torres Pacheco, E. (2014). Gestión educativa y su relación con la práctica docente en las instituciones educativas emblemáticas de la ciudad de Puno - 2014 - Perú.Comuni@ cción: Revista De Investigación En Comunicación Y Desarrollo, 6(1), 56-64. https://comunicacionunap.com/index.php/ rev/article/view/65/68

Valderrama, S. (2013). Pasos para elaborar proyectos de investigación científica. 2 da ed. Perú: San Marcos E. I. R. L., editor.

Vera, A. \& Blanco, A. (2019). Modelo para la gestión del talento humano en las pymes del sector servicios de Barranquilla, Colombia. Innovar, 29(74), 25-55. https://doi. org/10.15446/innovar.v29n74.82059

Villalobos, H. (2020). Technological Development in Police Matters: A Successful Recipe for Crime Prevention. Revista de Relaciones
Internacionales, Estrategia y Seguridad, 15(1), 79-97. https://doi.org/10.18359/ ries.4243

Willis, J., Koper, S. \& Lum, C. (2020) Technology use and constituting structures: accounting for the consequences of information technology on police organisational change. Policing and Society, 30(5), 483-501. https://doi.org/10.10 $\underline{80 / 10439463.2018 .1557660}$

Yépez, C. y Pozo, J. (2018). Gestión, Procesos y Calidad Universitaria: Caso Ecuatoriano. European Scientific Journal, 14(34), 195$217 . \quad$ https://doi.org/10.19044/esj.2018. v14n34p195

Zapata, G. \& Canet, M. (2012). Types of Work and Training for Task Specialization in the Organization. Revista de Ciencias Sociales, 18(1), 58-73. https://www. scopus.com/inward/record.uri?eid=2-s2.084864529321\&partnerID $=40 \& \mathrm{md} 5=350925$ cd328b3a17fadc990015b38ac1 